\title{
Buscando la clave de las elecciones del 94
}

\author{
Alvaro Artiga-González
}

Hacen lalta siete meses para que EI Salvador viva un proceso electoral excepcional; desde ya se le denomina:"LAS ELECCIONES DEL SIGLO". Y esto por varias razones, entre ellas:

- son las primeras elecciones en sesenta y cualro ańos, desde 1930 , en donde participarán todas las lendencias ideológicas representadas en los partidos políticos.

- son las primeras elecciones desde 1982 que se realizarán fuera del contexlo de guerra en el que se llevaron a cabo los nueve procesos electorales del periodo 1982-1991.

- desde 1982, año en que terminan los gobiernos de facto originados por el Golpe de Estado de Octubre de 1979, el proceso electoral de 1994 es el primero en el que, simullaneamente se dispulará y se distribuirá el "Poder Tolal" al elegirse Presidente de la República, Dipulados para la Asamblea Legislativa y Concejos Municipales.

En este contexio no es ocioso hacer una reflexión sobre el sistema electoral salvadoreño, entendiendo por ésle el procedimiento por el que los electores expresan su voluntad en votos y los votos, a su vez, se convierten en escafios.

$\mathrm{Si}$, refiriéndonos a las elecciones de dipulados a la Asamblea Legislativa, lo que se busca es una representación política proporcional, conviene que comenzemos estableciendo los parámetros de la discusión. 


\section{La normatlvidad del Codigo Electoral de 1993.}

En primer lugar, pueden ejercer el derecho al sufragio lodos aquellos ciudadanos, mayores de 18 anos, que eslén inscrilos en el Registro Electoral y que poseen su Carnet Elecloral.

En segundo lugar, el Artículo 10 del Código Electoral eslablece que habrán Ires lipos de circunscripciones electorales que corresponden a la división territorial en Municipios, Departamenlos y la Nación completa.

Para el caso de las elecciones para diputados, que son las que nos inleresan examinar en este trabajo, el Articulo 12 establece el tamano de las circunscripciones electorales, para un lotal de 84 escaños (puestos para dipulados), asi:

\begin{tabular}{|r|r|}
\hline Circunscripción Nacional: & 20 \\
\hline Circunscripiciones Departamentales: & \\
\hline San Salvador. & 16 \\
Sanla Ana & 6 \\
\hline San Miguel & 5 \\
La Libertad. & 5 \\
Usulután. & 4 \\
Sonsonate & 4 \\
La Unión & 3 \\
La Paz & 3 \\
Chalatenango. & 3 \\
Cuscatlán & 3 \\
Ahuachapán. & 3 \\
\hline Morazán & 3 \\
San Vicente & 3 \\
Cabanas & 3 \\
\hline Total & 84 escafios. \\
\hline
\end{tabular}

Finalmente, el Arlículo 262 del Código Electoral establece el procedimiento para distribuir los escanos entre los partidos polílicos en conlienda: el total de votos válidos para Diputados obtenidos en cada circunscripción se dividirá entre el número de Diputados propietarios que corresponda a esa circunscripción, obleniendo así el cociente electoral.

Una vez determinado dicho cociente, los Partidos Polílicos o Coaliciones tendrán tanlos diputados o escanos como veces esté contenido el cociente electoral en el número de votos que haya obtenido el Partido Político o Coalición en la circunscripción de que se Irale. 
$\mathrm{Si}$, luego de esta disıribución, existiesen escanos que asignar entre los Partidos Políticos o Coaliciones se eslablece una mecánica de Residuos Mayores:

"Si laltare un Dipulado que asignar, lo ganará el Partido Político o Coalición de mayor residuo; si fallaren dos, el segundo to ganara el Partido Político o Coalición que siga con mayor residuo y así sucesivamente hasla complementar el número de Diputados del Departamento.

Si uno o más Partidos Políticos o Coaliciones no alcanzaren el cocienle electoral se lomarán sus volos como residuos. Si ningún Partido Político o Coalición alcanzare el cociente elecloral, se adjudicará un Diputado a cada Partido Político o Coalición por el orden de mayoría de volos.

Cuando hubiere empate, en los casos de los dos incisos anteriores, el Dipulado lo ganará el Partido Polílico o Coalición que haya obtenido mayoria en el total de los residuos generales de toda la República"

Este procedimiento es válido lanto para las circunscripciones departamentales como para la circunscripción (la plancha) nacional.

2. Breves consideraclones teoricas sobre la constituclón de los sistemas politicos representatlvos.

Se ha dicho que el establecimiento de instiluciones parlamentarias, que tendrian por objelo original arrancar del monarca el control sobre el gobierno, es una innovación típica del siglo XIX (Rose,1983: p.76). En olras palabras, históricamente la representación nace para limitar el poder absoluto del soberano. Cuando el gobiemo es controlado por, o recae en, el soberano, el parlamento actuaria como representanle del pueblo. El poslerior desarrollo de esla institución le llevó a un status ambiguo: el parlamento aclua en un doble papel, como gobierno y como representante de los gobernados (Rodríguez,1987: p.137-189).

Los partidos políticos, como expresiones de la representación y participación política de los ciudadanos, siempre que no sean partidos antisislema (Sartori,1980: p. 167), tienen la intención explicita de participar en el gobierno o cuando menos formar parte de la oposición para iniluir desde alli sobre el gobiemo.

El mecanismo aceptado, deniro de los límites de la concepción democrática liberal, para dispularse el poder político lo constituyen las elecciones. A tal grado que se ha llegado a vincular estrechamente democracia con elecciones. Se dice, entonces, que alli dondo no hay elecciones no hay democracia. Por tanto, es a través de las elecciones como se establece la representación política de cualquier nación que se 
considere democrática.

Ahora bien, exislen dos principios alternativos de representación que establecen una distinción entre sistemas electorales (Nohlen,1984: pp. 24-25):

a. representación funcional/política:

- que da sentido a un sistema de mayoría absoluta o simple.

b. representación social/proporcional:

- que da sentido al sistema de representación proporcional de todas las fuerzas políticas de la nación.

A cada tipo de sislema electoral corresponde un tipo de fórmula de decisión para convertir los volos en escanos:

a. fórmulas de mayoria absolula/simple: el candidato o partido que obtenga la mayoría absoluta o simple de los votos válidos emitidos en una circunscripción elecloral determinada será elegido.

b. fórmulas de representación proporcional: los candidatos o partidos que hayan sido capaces de conseguir la cantidad necesaria de votos (cocienle o cuola elecloral) serán elegidos.

Es posible, sin embargo, que los sistemas electorales puedan combinar elementos de un sistema de mayoría simple y de representación proporcional al mismo tiempo (ibid:p.20).

Por otra parte, un sistema electoral proporcional no tija de antemano los porcentajes de represenlación a atribuir, tampoco cielermina el número de partidos que obtendrán representación en el parlamento. Aunque si fija un "lecho minimo" de votos para que el parlido o coalición sea considerado en la distribución de escańos 0 , como en el caso salvadoreho que se fija en el uno por ciento de los volos válidos, para que sea cancelado si no lo alcanzare.

Lo que se busca, en todo caso, es simplemente estimular - a diferencia de los sistemas mayoritarios - la presencia parlamentaria de más partidos, tendiendo a dificultar - aunque tampoco lo impide necesariamente- la aparición de uno o dos grandes partidos (Rose, 1983:p.93).

No obstante, y como decíamos arriba, es posible la existencia de efectos desproporcionales. Es decir, que los resultados electorales no se correspondan con el principio de representación proporcional. Esto es probable que ocurra en aquellos sistemas electorales en que se adjudican los escanos según una fórmula de representación proporcional en muchas circunscripciones de pequeno tamaño. El efeclo podría ser el de una representación por mayoría o de beneficio a los partidos grandes. 


\section{El sistema electoral salvedoreno "In actu".}

Es diflcil hacer un análisis a fondo de un proceso electoral que lodavia no se ha realizado. En especial si se trata del análisis de los resultados electorales. Por eso, el carácter de las siguientes consideraciones es hipolético pero no por ello carente de importancia. Vamos a utilizar las siguientes fuentes de dalos:

- resultados oficiales de las elecciones para diputados de 1991, publicados en la Revista ECA, No.509, Marzo 1991

- datos de la encuesta sobre lenencia de documentos de identificación personal, publicados por la Dirección General de Estadística y Censo (DGEC) el pasado 30 de Julio.

- datos muestrales del Censo de Población de 1992 publicados en La Prensa Gráfica, el 25 de Julio.

\subsection{Sobre la poblaclón representada.}

Tomando en consideración únicamente a la población salvadorefia que reside en el pais, tenemos que de un tolal de 5,047,925, los mayores de 18 anos que estarlan directamente representados en la Asamblea Legislativa serlan $2,700,430$, es decir, el $53.5 \%$ de la población. Según la DGEC, la población que tiene Cédula de Identidad Personal y que, por lanto, es mayor de 18 aftos es 2,739,473.

Ahora bien, según el código electoral, sólo votan los que tienen Carnet Electoral. Según la misma DGEC se trata de 1,957,325 los que to poseen a la fecha. Es decir, el $71.4 \%$ de los posibles votantes. Haria falta carnetizar a 782,148 personas $(28.6 \%)$. Una cantidad que puede ser decisiva para la conformación de la Asamblea.

Si tomamos como base la población estimada por la muestra censal, los resultados serlan:

Mayores de 18 anos:

$2,700,430$

Personas con carnet elecloral (DGEC): $\quad 1,957,325$

Personas a carnelizar:

$743,105(27.5 \%)$

Una cantidad que aunque menor que la estimada con base en los datos de la DGEC sigue siendo muy alta.

En las elecciones de 1991 se contabilizaron 1,051,481 votos válidos. Para ese evento se habian estimado 2,581,593 potenciales votantes (ECA No.509:p.228). Esto significa que 1,530,112 polenciales electores, o no votaron, 0 anularon su volo, o se abstuvieron. Aquella Asamblea 
habrla sido elegida por el $40.7 \%$ de los mayores de 18 anos y que tienen, por tanto, derecho a ser representados en el Organo Legislativo.

Si se mantiene ese comportamiento de no votar, anular o abstenerse en las elecciones Hel 94 podriamos tener:

Mayores de 18 anos:

$2,700,430$

Votos válidos en el 91 :

$1,051,481$

No votarian, anularian el voto o se abstendrían: $1,648,949$

es decir, el $61.1 \%$ de los electores potenciales. En otras palabras, la Asamblea Legislativa sería electa por el $39.9 \%$ de los mayores de 18 anos; tendria una representalividad de menos de la mitad de a los que la Constitución Política y el Codigo Electoral les establecen el derecho al sufragio. Además, una cifra de 1,051,481 votos válidos significaría apenas un $20.8 \%$ de la población lotal del país estimada por la mueslra censal que utilizamos para este trabajo, siendo que este porcentaje debería estar cerca del $53.5 \%$ tal como lo sehalamos al principio de este apartado.

Una grave responsabilidad se plantea enlonces a todas las fuerzas sociales y políticas, como al Tribunal Supremo Electoral, para elevar esos indices de representalividad parlamentaria. "LAS ELECCIONES DEL SIGLO" no lo pueden ser si dan como resultado un bajo porcentaje no sólo de participación sino de volos válidos.

Una amplia movilización social es necesaria no solo para carnetizar sino para persuadir al electorado para que haga un uso eficaz del derecho al sufragio. De todas maneras, los futuros diputados legislarán para toda la nación, no importará si quienes les eligen consliluyen un 25 o 30 por ciento de la población. Deben superarse entonces problemas lécnicos y políticos (sobre lodo) para lograr una "masiva" participación electoral, y promoverse una nueva forma de hacer política, una nueva cullura política, en el pais para que esa participación se convierta en votos válidos.

\subsection{Sobre la transformación de votos en escafios.}

Vamos a utilizar el cuadro No. 1 elaborado con base en los resultados electorales de 1991. Como se sabe, el cociente elecloral es el número de votos que necesila un partido político o coalición para ganar un dipulado. Este cuadro nos mueslra algo que no es nuevo pero sí importante enlatizarlo: los votos válidos, en la pequenez territorial salvadorena, no tienen el mismo valor. Se argumenlaría que dentro de la misma circunscripción electoral si to liene, y sería correcto. Pero de circunscrip- 
ción a circunscripción no lo es $y$, aunque los diputados provengan de distintas circunscripciones legislarán para lodo el pais, y muy rara vez para la circunscripción en la cual salieron electos.

\section{Cuadro No.1:}

Cociente electoral (Volos/\#escartos) por Departamento en 1991.

\begin{tabular}{lrrr}
\hline Circunscripción & Tamano & Votos Válidos & Coc. Elec. \\
\hline San Salvador & 16 & 326,329 & $20,395.56$ \\
Santa Ana & 6 & 104,948 & $17,491.33$ \\
San Miguel & 5 & 68,489 & $13,697.80$ \\
La Libertad & 5 & 115,328 & $23,065.60$ \\
Usulután & 4 & 57,199 & $14,299.75$ \\
Sonsonate & 4 & 87,129 & $21,782.25$ \\
La Unión & 3 & 37,037 & $12,345.67$ \\
La Paz & 3 & 55,709 & $18,569.67$ \\
Chalatenango & 3 & 37,663 & $12,554.33$ \\
Cuscallán & 3 & 35,938 & $11,979.33$ \\
Ahuachapán & 3 & 51,211 & $17,070.33$ \\
Morazán & 3 & 23,930 & $7,976.67$ \\
San Vicente & 3 & 29,260 & $9,753.33$ \\
Cabanas & 3 & 21,311 & $7,103.67$ \\
\hline Totales & 64 & $1,051,481$ & \\
\hline
\end{tabular}

Fuente: Resultados electorales publicados en ECA No.509, Marzo de 1991.

Veamos el caso de Usulután, La Paz y Ahuachapán. Se necesitaron menos volos para ganar un diputado en Usulután que para ganarlo en La Paz y Ahuachapán. El volo de Usululán valdría más que en La Paz y Ahuachapán. Pero, además, el primero liene asignados cuatro escahos frente a tres de los últimos. Es decir, con los volos de Usulután se ganan más dipulados que con los volos en La Paz y Ahuachapán considerados individualmente.

El mismo resullado enconlramos entre San Miguel, Usulután y Sonsonate. $Y$ todavia se necesitaron más volos en La Paz y Ahuachapán para ganar un escafio frente a San Miguel. Es decir, con menos volos para ganar un escano en esle último deparlamenlo se tienen más diputados que en los otros cualro deparlamentos (5 contra 4 y 3 respectivamente). 
Para las estrategias electorales de los partidos políticos, hechos como esle, pueden resullar importantes, hasta pueden signiticar una especie de "carla escondida". Asi es como se podrla conseguir una "mayorla parlamentaria manufacturada". El votante podría entonces plantearse la pregunta: ¿qué hace el sistema electoral con mi volo?

Por otra parte, Sonsonate tuvo más volos válidos que San Miguel, y La Libertad más votos válidos que Santa Ana. Sin embargo, tanto Sonsonate como La Libertad tienen un escafio menos que San Miguel y Santa Ana, respectivamente. Cantidades mayores se dividen entre cantidades menores dando como resultado cocientes electorales mayores para los primeros. Es decir, se necesitan más volos para lograr un escano. ¿A quién se favorece: a los partidos grandes o a los partidos pequenos? Aqul es donde es determinante el tamano de la circunscripción y el número de partidos en la compelencia. En circunscripciones grandes, la ventaja podrla ser para los partidos pequenos (cuando se recurre a la mecánica de los residuos o restos mayores). En circunscripciones pequenas, los partidos mayores podrian sacar ventaja. Si tomamos en cuenta los cerca de 750 mil nuevos volantes, manteniendo el mismo número de escanos, la canlidad de volos por escano será mayor, favoreciéndose así los partidos mayores.

\section{Cuadro No.2:}

Población total, mayor de 18 años y con carnet electoral por departamento o circunscripción electoral.

\begin{tabular}{lrrrrrrr}
\hline Circunscripción & Tamaño & $\%$ & Habilantes & \multicolumn{1}{c}{$>$ 18 años } & $\%$ & c.Carnet & $\%$ \\
\hline San Salvador & 16 & 25.0 & $1,477,766$ & 790,605 & 29.27 & 648,563 & 33.14 \\
Santa Ana & 6 & 9.38 & 451,620 & 241,617 & 0.95 & 184,197 & 9.41 \\
San Miguel & 5 & 7.80 & 380,442 & 203,536 & 7.54 & 129,431 & 6.61 \\
La Libertad & 5 & 7.80 & 522,071 & 279,308 & 10.34 & 195,465 & 9.99 \\
Usulután & 4 & 6.25 & 317,079 & 169,637 & 6,28 & 113,879 & 5.82 \\
Sonsonate & 4 & 6.20 & 354,641 & 189,733 & 7.03 & 142,597 & 7.27 \\
La Unión & 3 & 4.69 & 251,143 & 134,362 & 4.98 & 76,575 & 3.91 \\
La Paz & 3 & 4.69 & 246,147 & 131,689 & 4.88 & 79,956 & 4,08 \\
Chalatenango & 3 & 4.69 & 180,627 & 96,635 & 3.58 & 60,935 & 3.11 \\
Cuscatlán & 3 & 4.69 & 167,290 & 89,500 & 3.31 & 63,471 & 3.24 \\
Ahuachapán & 3 & 4.69 & 260,563 & 139,401 & 5.16 & 113,161 & 5.78 \\
Morazán & 3 & 4.69 & 166,772 & 89,223 & 3.30 & 56,773 & 2.90 \\
San Vicente & 3 & 4.69 & 135,471 & 72,477 & 2.68 & 47,353 & 2.43 \\
Cabañas & 3 & 4.69 & 136,293 & 72,917 & 2.70 & 44,979 & 2.31 \\
\hline Totales & 64 & 100.00 & $5,047,925$ & $2,700,640$ & 100.00 & $1,957,325$ & 100.00
\end{tabular}

Fuente: Muestra Censal y Encuesta DGEC. 
Vamos a considerar ahora únicamente las circunscripciones departamentales para observar la proporcionalidad de estas Irente al total de habitantes, la población mayor de 18 ahos y la población cametizada a la lecha.

Lo primero que salta a la vista es que la circunscripción elecloral de La Liberlad, pese a lener una mayor proporción de habitantes y ciudadanos mayores de 18 anos, liene asignada una menor proporción parlamentaria comparada con Santa Ana.

Ahuachapán liene casi el doble de población, lotal y mayor de 18 anos, que San Vicente y Cabahas, no obstante tiene asignados igual cantidad de escanos. Fuera de estos casos, los datos muestran cierta tendencia del tamano de la circunscripción a representar proporcionalmente la distribución lerritorial de la población. Pero aquí es donde cabe la pregunta: ¿se trata de una proporcionalidad de la distribución terrilorial de la población o, mas bien, de la proporcionalidad de la fuerza de los partidos políticos?

Véase que eslos aspectos sehalados refuerzan no solo la desigualdad en el valor del voto sino que también la posibilidad de "manulacturar mayorias". Este hecho no es tan hipotélico que se diga. El cuadro No.3 nos muestra la distribución de los 64 escanos de las circunscripciones departamentales en las elecciones de 1991:

Cuadro No.3:

Distribución de diputados en las elecciones legislativas del 10 de marzo de 1991.

\begin{tabular}{lccccccc}
\hline Circunscripción & ARENA & PDC & PCN & MAC & CD & UDN & TOTAL \\
\hline San Salvador & 7 & 4 & 1 & - & 3 & 1 & 16 \\
Sanla Ana & 3 & 2 & - & - & 1 & - & 6 \\
San Miguel & 2 & 2 & 1 & - & - & 1 & 5 \\
La Libertad & 3 & 1 & - & - & 1 & - & 5 \\
Usulután & 2 & 1 & 1 & - & - & - & 4 \\
Sonsonate & 2 & 1 & 1 & - & - & - & 4 \\
La Unión & 1 & 2 & - & - & - & - & 3 \\
La Paz & 2 & 1 & - & - & - & - & 3 \\
Chalatenango & 1 & 1 & - & - & 1 & - & 3 \\
Cuscatlán & 2 & 1 & - & - & - & - & 3 \\
Ahuachapán & 1 & 1 & 1 & - & - & - & 3 \\
Morazán & 1 & 1 & 1 & - & - & - & 3 \\
San Vicente & 2 & 1 & - & - & - & - & 3 \\
Cabañas & 1 & 1 & 1 & - & - & - & 3 \\
\hline Totales & 30 & 20 & 7 & - & 6 & 1 & 64 \\
\hline
\end{tabular}

Fuente: ECA No.509, Marzo 1991.p. 158. 
Claramente se observa que ARENA, PDC y PCN se repartieron siete de las ocho circunscripciones de 3 escahos. Si tomamos en cuenta la poca distancia ideológica (Sartori, 1980:pp.102-112) entre ARENA y PCN observamos cómo las pequefias circunscripciones habrían lavorecido, en este caso, una "mayoría de derecha". Es decir, con circunscripciones electorales pequehas exisle la posibilidad de que el sistema electoral, pretendido proporcional, produzca resultados de un sistema de mayoría simple.

Por otra parte, las circunscripciones grandes, como la de San Salvador, dieron un resullado más proporcional y si se lo quiere ver, considerando la "distancia ideológica" entre los partidos se tiene un empate a ocho entre ARENA-PCN y PDC-CD-UDN.

Se han sefialado ya algunas siluaciones que ocurren en la distribución de escanos al hablar de las circunscripciones eleclorales. Vamos a referirnos ahora a los resullados concretos que ha producido, y puede producir en el próximo evento electoral, la utilización de la mecánica de los restos o residuos mayores y que tienen que ver $\infty$ n la proporcionalidad prelendida por el sislema elecloral salvadorefio.

\section{Cuadro No.4:}

Volos válidos y número de diputados por partido en las elecciones de 1991.

\begin{tabular}{lrrrrr}
\hline Partido & Volos Válidos & $\%$ & No.Dipul. & \multicolumn{1}{c}{$\%$} \\
\hline ARENA & 466,091 & 44.33 & 39 & 46.43 \\
PDC & 294,029 & 27.96 & 26 & 30.95 \\
PCN & 94,531 & 8.99 & 9 & 10.71 \\
MAC & 33,971 & 3.23 & 1 & 1.19 \\
CD & 127,855 & 12.16 & 8 & 9.53 \\
AD & 6,798 & 0.65 & - & - \\
UDN & 28,206 & 2.68 & 1 & 1.19 \\
\hline Totales & $1,051,481$ & 100.00 & 84 & 100.00 \\
\hline
\end{tabular}

Fuente: ECA No.509, Marzo 1991: pp.158 y 234.

Lo primero que salta a la visla del Cuadro No. 4 es que el PCN, con menos volos válidos obtuvo más diputados que Convergencia Democrática. Por otra parte, los dos grandes partidos (ARENA y PDC) tienen el $77.38 \%$ de los escanos. Si los 84 dipulados se disputaran en una cir- 
cunscripción nacional, los resultados hubieran sido, lomando en cuenta un cociente electoral de 1,051481/84 $=12,517.63$ los mostrados en el Cuadro No. 5.

Las diferencias entre la proporción de volos y la proporción de diputados por partido son más pequehas que las que se pueden deducir de los datos moslrados en el Cuadro No.4. Por tanlo, la distribución de dipulados reflejarla mejor la distribución de los votos. Utilizando dos tipos de circunscripción electoral (nacional y departamental), con grandes diferencias entre si en relación a su tamano, se ha favorecido a los dos partidos mayores en delrimenlo de los partidos politicos menores.

\section{Cuadro No.5:}

Votos válidos y número de dipulados por partido en las elecciones del 91 .

si se hubieran dispulado en una sola circunscripción electoral nacional.

\begin{tabular}{|c|c|c|c|}
\hline rlido & V.Válidos & & \\
\hline
\end{tabular}

\begin{tabular}{|c|c|c|c|c|c|c|c|}
\hline ARENA & 466,091 & 44.33 & 37 & $2,938.69$ & - & 37 & 44.05 \\
\hline PDC & 294,029 & 27.96 & 23 & $6,123.51$ & - & 23 & 27.38 \\
\hline PCN & 94,531 & 8.99 & 7 & $6,907.59$ & 1 & 8 & 9.53 \\
\hline MAC & 33,971 & 3.23 & 2 & $8,935.74$ & 1 & 3 & 3.57 \\
\hline$C D$ & 127,855 & 12.16 & 10 & $2,678.70$ & - & 10 & 11.90 \\
\hline$A D$ & 6,798 & 0.65 & - & $6,798.00$ & 1 & 1 & 1.19 \\
\hline UDN & 28,206 & 2.68 & 2 & $3,170.74$ & - & 2 & 2.38 \\
\hline & & & & & 3 & 84 & 100.00 \\
\hline
\end{tabular}

Fuente: ECA No.509,Marzo 1991:p.234.

Por otra parte, el Cuadro No.5 relleja cómo a mayor cantidad de volos más dipulados logran los partidos. Hecho que ya advertíamos no sucede en el Cuadro No.4 para el caso PCN-CD. Sin considerar los diputados de la "Plancha Nacional", la distribución de diputados en 1991 hubiese sido la mosirada en el Cuadro No.6. Vemos que es aqui precisamente donde se da el efecto desproporcional entre PCN y CD. Con un $8.99 \%$ de los votos, el PCN tiene un diputado más que la CD a pesar de que ésta tiene un $12.6 \%$ de los votos válidos. 
Cuadro No.6:

Distribución de diputados en 1991, tomando en consideración sólo las circunscripciones departamentales.

\begin{tabular}{lrcc}
\hline Partido & \%votos & \#Dipulados & \%Diputados \\
\hline ARENA & 44.33 & 30 & 46.87 \\
PDC & 27.96 & 20 & 31.25 \\
PCN & 8.99 & 7 & 10.94 \\
MAC & 3.23 & - & - \\
CD & 12.16 & 6 & 9.38 \\
AD & 0.65 & - & - \\
UDN & 2.68 & 1 & 1.56 \\
\hline Totales & 100.00 & 64 & 100.00 \\
\hline
\end{tabular}

Fuente: ECA No.509, Marzo 1991:pp.158 y 234.

\section{En resumen:}

Si, teóricamente, la mecánica de los residuos o reslos mayores favorecerla a los partidos pequehos, en el caso salvadoreho vemos que son los partidos mayores los que se benefician. La explicación de este hecho está no en una falla de la mecánica de reparto sino más bien en el tamano de las circunscripciones electorales (que dicho sea de paso, hemos vislo no corresponden en todos los casos al tamano de la población que habita en cada circunscripción elecloral departamental). Es cierto que la composición final de la Asamblea Legislaliva lambién depende del sistema de partidos (objeto de estudio que dejamos para otra ocasión). Pero de cara a las elecciones del 94 podemos plantear como hipótesis que, dada la misma dislribución de escanos en las circunscripciones electorales que para 1991, y dado un aumento en la población de votanles, van a ser los partidos mayores los que se beneliciarán del sistema electoral salvadoreno. Sobre todo si consideramos que 24 escafios se disputan en 8 circunscripciones eleclorales. La "distancia ideológica" entre algunos partidos políticos volverá a ser clave para producir una "mayoria manulacturada" y controlar de esa manera el órgano que ha de legislar para el Eslado salvadoreno hasta 1997.

San Salvador, Agosio de 1993. 


\section{Referenclas}

Asamblea Legislativa (1993). "Código electoral 1993". San Salvador: fotocopia. DGEC (1993). "Encuesta sobre tenencia de Documentos de kdentificación Personal". San Salvador: lolocopia.

La Prensa Gráfica (1993). Población salvadoreña es de jóvenes y la mayoría son mujeres", p.21. San Salvador.

Nohlen, Dieter (1984). "Los sistemas electorales entre la ciencia y la ficción. Requisitos históricos y tebricos para una discusión racional", en Revista de Estudios Pollticos (Nueva Epoca),No.42,pp.7-31. Madrid: Centro de Estudios Constitucionales.

Rodrlguez D., Angel (1987). "Un marco para el análisis de la representación politica en los sistemas democráticos" en Revista de Estudios Polfticos (Nueva Epoca), No.58,pp. 137-190. Madrid: Centro de Estudios Constitucionales.

Rose, Richard (1983). "En torno a las opciones en los sistemas electorales: alternativas politicas y lócnicas" en Revista de Estudios Politicos (Nueva Epoca), No.34,pp.60-105. Madrid: Centro de Estudios Constitucionales.

Sartorio, Giovanni (1980). "Partidos y sistemas de partidos". Madrid, Alianza Editorial, S.A.

UCA (1991). "Revista Estudios Centroamericanos (ECA), No.509, Marzo 1991". San Salvador: Universidad Centroamericana "José Sim -hn Cañas". 